\title{
Review Article \\ Squamous cell carcinoma of the head and neck region: Insights from the American Society of Clinical Oncology 2008 presentations
}

\author{
Desai C \\ Hemato-Oncology Clinic, Vedanta Institute of Medical Sciences, Navrangpura, \\ Ahmedabad - 380 009. India.
}

Correspondence to: Dr. Chirag Desai, E-mail: chiragdesai.oncology@gmail.com

\section{Introduction}

Squamous cell carcinoma of the head and neck region (H N SCC) remains an important problem for the majority of countries of the developing world. Most patients present with advanced disease. $\mathrm{H}$ ence, it is a challenge to improve cure rates as well as to provide meaningful survival advantage to the others. In India alone, it is estimated that more than 2.5 lakh new patients are diagnosed every year, of whom about three-fourths are in an advanced stage. The population-based cancer registry of the Indian Cancer Society shows that the number of patients with H NSCC is increasing significantly.

As per the Amercian $\mathrm{N}$ ational Comprehensive Cancer N etwork ( NCCN) guidelines (Version 2, 2008), the standard of care for patients with locally advanced H NSCC is concomitant chemoradiotherapy (CRT). This is either used directly or preceded by induction combination chemotherapy. H owever, a significant number of patients do not tolerate such an aggressive approach. This is important for our patients with HNSCC because many of them are already malnourished, have impaired food intake and are dehydrated - features that would compromise their ability to tolerate CRT. The Bonner study addressed just this challenge. ${ }^{[1]}$ In this prospective randomized phase III trial, a total of 211 patients were treated with a combination of cetuximab $\left(E_{\text {rbitux }}{ }^{\circledR}\right.$ ) and radiotherapy (RT) study arm versus the control arm of RT alone. The addition of cetuximab increased both locoregional control as well as overall survival (OS). The median duration of locoregional control in the cetuximab arm was 24.4 months as compared with 14.9 months with RT alone (P-value 0.005). The OS was almost doubled (49.0 months) by adding cetuximab (as compared with 29.3 months with RT alone; P-value 0.03 ). A nother study by Pfister et al. provides additional data regarding consistency of cetuximab benefit. ${ }^{[2]} \mathrm{H}$ ere, patients received concomitant $\mathrm{RT}$, cisplatin and cetuximab. In patients with stage III or IV (without distant metastasis) HNSCC, at a follow-up of 52 months, this combination gave a 3 -year OS of $76 \%$ and a 3-year locoregional control rate of $71 \%$. Such dramatic improvement led to inclusion of cetuximab as standard of care in the British $\mathrm{N}$ ational Institute of $\mathrm{H}$ ealth and Clinical Excellence (NICE) guidelines (issued in June 2008). NICE works as an independent organization responsible for providing national guidance on the promotion of good health and the prevention and treatment of ill health. It enjoys a worldwide reputation, especially because it uses the expertise of the British $\mathrm{N}$ ational $\mathrm{H}$ ealth Services to develop and validate its guidelines.

\section{American Society of Clinical Oncology 2008 Update}

An important highlight of the American Society of Clinical Oncology (ASCO) 2008 in Chicago was the multitude of abstracts on the management of H NSCC. There were as many as 81 presentations on this topic. Of significance was the additional data on the role of cetuximab for this indication.

In recent times, cetuximab has earned the stamp of consistent patient benefit across disease indications. For H N SCC, the abstracts included neoadjuvant/induction therapy, concurrent therapy as well as use in the adjuvant setting. All the abstracts put together representative data from a total of over 500 patients (excluding those with nasopharyngeal carcinoma). This is more than twice the number of cases in the Bonner study $(n=211) .^{[1]}$ Let us, therefore, take this opportunity to discuss important lessons to be learned from its interpretation.

Benefit of dose intensity: Gilliham et al. reported a single-institution experience with RT and concomitant cetuximab in locally advanced H NSCC wherein the 


\begin{tabular}{|c|c|c|c|c|}
\hline Patient characteristics & RT/CDDP (n = 34) & $\mathrm{RT} / \mathrm{CTX}(\mathrm{n}=34)$ & $\begin{array}{l}\text { RT with concomitant } \\
\text { boost/CTX }(n=14)\end{array}$ & $\begin{array}{c}\text { Bonner trial } \\
(n=211)\end{array}$ \\
\hline Median age (range) & $58(29-79)$ & $63(44-82)$ & $61(39-76)$ & $56(34-81)$ \\
\hline RT dose (Gy) & $66-70$ & $66-70$ & 70 & $70-72$ \\
\hline$\geq \mathrm{G} 3$ skin toxicity (\%) & 18 & 58 & 86 & 23 \\
\hline$\geq \mathrm{G} 3$ oral toxicity (\%) & 44 & 78 & 77 & 56 \\
\hline$\geq \mathrm{G} 3$ acneiform rash (\%) & 0 & 11 & 4 & 17 \\
\hline Compliance with treatment $(\%)^{*}$ & 59 & 79 & 86 & Not available \\
\hline
\end{tabular}

\begin{tabular}{|c|c|c|c|c|c|c|c|c|}
\hline \multirow{2}{*}{$\begin{array}{l}\text { Patients with grade } 3 / 4 \text { AEs (\%) } \\
\text { Maintenance phase }\end{array}$} & \multicolumn{2}{|c|}{ End of RT } & \multicolumn{2}{|c|}{ Week 4} & \multicolumn{2}{|c|}{ Week 8} & \multicolumn{2}{|c|}{ Week 12} \\
\hline & No & Yes & No & Yes & No & Yes & No & Yes \\
\hline Acne-like rash & 8.9 & 8.5 & 6.6 & 4.3 & 0 & 2.1 & 0 & 0 \\
\hline Mucositis & 40 & 55 & 6.7 & 10.6 & 0 & 4.3 & 0 & 2.1 \\
\hline Radiation dermatitis & 17.8 & 4.3 & 15.6 & 14.9 & 0 & 0 & 0 & 0 \\
\hline Asthenia & 0 & 4.3 & 0 & 2.1 & 0 & 0 & 2.2 & 0 \\
\hline
\end{tabular}

acute toxicity data was recorded prospectively using the Common Terminology Criteria version 3.0 questionnaire. [3] In spite of the expected increase in skin and oral toxicity with cetuximab, the overall treatment compliance was better [ $79 \%$ versus $59 \%$ with RT/cisplatin (CDDP); $P$-value 0.003] and septic episodes were lesser ( $4 \%$ versus $12 \%$ with RT/CDDP; P-value 0.04$)$. D etails are shown in Table 1.

The Spanish multicentric prospective randomized study of concomitant RT and cetuximab with or without maintenance cetuximab gives important confirmation of its safety and efficacy. ${ }^{[4]}$ The dose intensity was more than $85 \%$ for both the concomitant part as well as the maintenance part of the protocol. The grade 3 and 4 toxicities are shown in Table 2. By week 12, the patients receiving maintenance cetuximab showed only a slightly higher incidence of mucositis, which was balanced by the higher asthenia in the nonmaintenance arm.

Preliminary analysis of ECOG 3303 data on concurrent chemoradiotherapy (cetuximab+cisplatin + RT) confirms the safety of this combination. ${ }^{[5]}$ Grade 3 and 4 toxicities were manageable and less than expected $26 \%$ neutropenia, $28 \%$ acne-form rash, $23 \%$ fatigue, $15 \%$ radiation dermatitis and $54 \%$ mucositis. Therefore, among the 61 evaluable patients, $87 \%$ of the patients could receive all the planned treatments.

For concomitant hyperfractionated accelerated RT with cisplatin and cetuximab, Kuhnt et al. showed an objective remission rate (ORR) of 91\% ( $n=14$ patients) without any deaths. ${ }^{[6]}$

N eoadjuvant/induction setting: The $\mathrm{N}$ ew England J ournal of Medicine article of 2007 has established that in locally advanced H NSCC, use of neoadjuvant TPF (docetaxel+cisplatin + 5-FU) followed by RT or CRT improves OS as well as organ preservation. ${ }^{[7]}$

The retrospective analysis from Lyon, France, indicated that neoadjuvant TPF resulted in $23 \%$ grade $3 / 4$ neutropenia and $14 \%$ febrile neutropenia in the first cycle in spite of prophylactic growth factor use. ${ }^{[8]}$ For those patients who went on to undergo combination $\mathrm{RT}$, treatment interruption due to toxicity remained a major problem compromising dose intensity. There was permanent discontinuation of systemic therapy in $69 \%$ of the cases in the combination cisplatin arm as opposed to only a $28 \%$ permanent interruption in the concomitant cetuximab arm. This indicates that concomitant cetuximab is better tolerated and allows a greater percentage of patients to receive a proper dose intensity - a factor that is directly linked to better OS.

Induction TPE (docetaxel + cisplatin + cetuximab) followed by concurrent CRT shows a promising survival benefit. ${ }^{[9]}$ The ORR for the TPE part was $86 \%$ [32/37; with $13 / 22$ (59\%) showing CR on PET scan]. Following the completion of the CRT part of the treatment, the ORR increased to $100 \%$ [22/22; with $19 / 22$ (86\%) showing CR on PET scan]. At a 
median follow-up of 12 months, only four patients had died (three due to disease progression and one due to myocardial infarction).

Recurrent/metastatic setting: In the recurrent/ metastatic setting of platinum-pretreated patients, use of docetaxel and cetuximab combination was reported by $\mathrm{K}$ noedler et al. in a multicentric phase II study from Germany. ${ }^{[10]}$ This is a group of patients with extremely poor prognosis. Among the 45 evaluable patients, they found an overall disease control rate of $47 \%$. This response was possible with grade 3/4 skin toxicity in nine cases and other grade $3 / 4$ toxicity in 10 patients.

Reducing toxicity: Tra et al. reported the use of albuterol-based premedication to prevent cetuximab infusion-related reactions. ${ }^{[11]}$ All patients receiving cetuximab (from February 2004 onwards) received premedication with IV diphenhydramine $25 \mathrm{mg}$, oral acetaminophen $1000 \mathrm{mg}$, IV ondansetron $8 \mathrm{mg}$ and inhalation albuterol [abluterol/ipatropium (Duoneb) or levalbuterol (Xopenex); 0.63-1.25 mg) via nebulizer 30 min before cetuximab infusion. $N$ ot a single one of the approximately 100 patients studied developed any cetuximab infusion reaction. This is in contrast to a 3\% incidence reported in patients not receiving albuterolbased premedication.

For concomitant cetuximab and TPF (docetaxel, cisplatin, 5-FU), the gastrointestinal toxicity is dose limiting and can be addressed by reducing the dose of 5-FU to $850 \mathrm{mg} / \mathrm{m}^{2}$. ${ }^{[9]}$ As far as cetuximab is concerned, the only toxicity that limits dosing is the skin rash.

Indian context: In India, the most common therapy used for locally advanced H NSCC is CRT with cisplatin. The data presented at ASCO 2008 indicate that cetuximab can be added to the above protocol or may replace cisplatin. Although some toxicities are expected that are typical for cetuximab (especially skin and mucosal), the actual dose intensity that can be administered to the patient is higher due to the lack of typical side effects of cisplatin. H ence, the addition of cetuximab should improve the ORR as well as survival.

Dose intensity and, therefore, efficacy can be enhanced by proper selection of patients, judicious use of coprescriptions as well as ensuring adequate nutrition and hydration.

\section{Conclusion}

The problem of H NSCC is increasing in our country. With the majority of patients having locally advanced disease, optimal management remains a challenge. U se of CRT is the standard of care in such cases. Cetuximab offers consistent benefit in several cancers, including H NSCC. Its use with RT or with CRT has proven to improve ORR as well as survival. As a result, it has now been included in NICE, the UK recommendations. ASCO 2008 included additional data from over 500 patients. Simple measures will ensure that your patients will benefit from the advantages of cetuximab - particularly by ensuring dose intensity with acceptable toxicity.

Thus, current data on cetuximab supports its use in patients with HNSCC - as single agent, in combination with RT as well as a substitute for or in combination with platinum chemotherapy.

\section{References}

1. Bonner JA, Harari PM, Giralt J, Azarnia N, Shin DM, Cohen RB, et al. Radiotherapy plus cetuximab for squamous-cell carcinoma of the head and neck. N Engl J Med 2006;354:567-78.

2. Pfister DG, Su YB, Kraus DH, Wolden SL, Lis E, Aliff TB, et al. Concurrent cetuximab, cisplatin, and concomitant boost radiotherapy for locoregionally advanced, squamous cell head and neck cancer: A pilot phase II study of a new combined-modality paradigm. J Clin Oncol 2006;24:1072-8.

3. Gillham C. Toxicity of cetuximab and radiotherapy in locally advanced head and neck cancer: a community-based experience. J Clin Oncol; 26:6020.

4. Mesia R. Safety report of a randomized phase II trial to evaluate the combination of cetuximab plus accelerated concomitant boost radiotherapy (RT) followed or not followed by cetuximab monotherapy in patients (pts) with locally advanced squamous cell carcinoma of the oropharynx. J Clin Oncol; 26:6076.

5. Langer CJ. Preliminary analysis of ECOG 3303: Concurrent radiation (RT), cisplatin (DDP) and cetuximab (C) in unresectable, locally advanced (LA) squamous cell carcinoma of the head and neck (SCCHN). J Clin Oncol; 26:6006.

6. Kuhnt T. Concomitant hyperfractionated accelerated radiotherapy (HART) with cisplatin and concurrent cetuximab for locoregionally advanced squamous cell head and neck cancer: a phase I dose escalation trial. J Clin Oncol; 26:6029.

7. Vermorken JB, Remenar E, van Herpen C, Gorlia T, Mesia R, Degardin $\mathrm{M}$, et al. Cisplatin, fluorouracil, and docetaxel in unresectable head and neck cancer. N Engl J Med 2007;357: 1695-704.

8. Bonnin N. Efficacy of neoadjuvant TPF (nTPF; docetaxel, T; cisplatin, $\mathrm{P} ; 5 \mathrm{FU}$ ) in nonselected patients (pts) with head and neck cancer and subsequent radiotherapy (RT) combined with chemotherapy (CT) or cetuximab (Cx). J Clin Oncol; 26:6074.

9. Argiris AE. Phase II trial of neoadjuvant docetaxel (T), cisplatin $(P)$, and cetuximab $(E)$ followed by concurrent radiation $(X), P$, and $E$ in locally advanced head and neck cancer (HNC). J Clin Oncol; 26:6002.

10. Knoedler M. Phase II trial to evaluate efficacy and toxicity of cetuximab plus docetaxel in platinum pretreated patients with recurrent and/or metastatic head and neck cancer. J Clin Oncol; 26:6066

11. Tra F, Fesen M, Nisty R. Albuterol-based premedication therapy for the prevention of cetuximab infusion-related reactions. J Clin Oncol; 26:17040.

Source of Support: Nil, Conflict of Interest: None declared. 